Neurosurg Focus 11 (6):Article 7, 2001 Click here to return to Table of Contents

\title{
Stereotactic intensity-modulation radiation therapy for vertebral body and paraspinal tumors
}

\author{
Mark H. Bilsky, M.D., Kamil Yenice, M.D., Michael Lovelock, Ph.D., \\ AND JOSH YAMADA, M.D. \\ Departments of Surgery, Medical Physics, and Radiation Oncology, Memorial Sloan-Kettering \\ Cancer Center, New York, New York
}

\begin{abstract}
Therapeutic doses of radiation for paraspinal tumors are often limited by the dose-related tolerance of the spinal cord. Intensity-modulated radiation therapy (IMRT) is an advanced form of three-dimensional conformal radiation therapy that provides improved coverage of tumor volumes while reducing the radiation dose to the spinal cord. Computer-controlled multileaf collimation provides high conformality, which makes it feasible to treat tumors of any shape, even those that are wrapped around the spinal cord. The use of a newly developed, noninvasive body frame, the capability of fusing computerized tomography and magnetic resonance images, and on-line portal films provide precise target immobilization and target identification. In this paper the authors discuss their preliminary experience in six cases in which IMRT was used to treat paraspinal lesions in patients who harbored locally recurrent tumors and/or tumors that previously received the maximum doses of radiation that could be tolerated by the spinal cord.
\end{abstract}

\section{KEY WORDS • stereotaxis • radiosurgery • intensity-modulated radiation therapy • spinal lesion}

Stereotactic radiotherapy has been used to treat intracranial tumors with great success over the past decade, but the application of this technology to extracranial sites has been limited by the need to develop a reproducible bodyimmobilization frame, target identification, and conformality to a variety of tumor shapes. Intensity-modulated radiation therapy represents an advanced form of 3D conformal radiotherapy in which multileaf collimators are used to change the field shape dynamically during treatment, thus permitting the delivery of an inhomogeneous radiation dose that conforms tightly to the target region. Using IMRT, an optimized radiation treatment plan can be devised that allows delivery of multiple intensity-modulated fields in 3D space to generate highly conformal tumor dose while sparing the maximum amount of normal tissue. At MSKCC, this technology was initially applied

Abbreviations used in this paper: $\mathrm{CT}=$ computerized tomography; IMRT $=$ intensity-modulated radiation therapy; MR = magnetic resonance; MSKCC $=$ Memorial Sloan-Kettering Cancer Center; $3 \mathrm{D}=$ three-dimensional. in the treatment of patients with prostate cancer in whom the tumor could tolerate a high (8100-cGy) therapeutic dose while limiting toxic doses to the adjacent bowel, rectum, urethra, and bladder. ${ }^{7}$

Paraspinal tumors without significant epidural compression (for example, those of the vertebral body, posterior element, and chest wall) represent another treatment paradigm that may benefit from IMRT technology. In standard external-beam radiotherapy of the spine, in which a single posterior field or opposed fields encompassing the involved segment are used, a therapeutic dose often cannot be delivered without causing catastrophic radiation-induced myelopathy; thus many tumors are suboptimally treated, and high recurrence rates result. Attempts to deliver high-dose radiation to the spinal canal, such as brachytherapy, have been hindered by significant technical limitations. ${ }^{3,6}$ Using IMRT technology, paraspinal tumors can be treated to a therapeutic dose while the spinal cord and adjacent structures (lung, bowel, and salivary glands) are spared toxic ( $>4500-\mathrm{cGy})$ radiation doses. ${ }^{1,2,4,5}$ The shape of the tumor does not restrict treatment, as is the case in standard photon stereotactic radio- 
surgery units currently used to treat intracranial tumors. Intensity-modulated radiation therapy establishes a dose gradient between the target and spinal cord that allows one to treat any tumor shape, even those that wrap around the spinal canal. Perhaps the greatest challenge in the development and application of the new technology has been target identification and rigid, reproducible body immobilization.

\section{INTENSITY-MODULATED RADIATION THERAPY}

\section{Application of the Technology}

Intensity-modulated radiation therapy in which dynamic multileaf collimation is used is a powerful tool that enables the safe delivery of high doses of conformal radiation. This modality depends on two factors: 1) computerized iterative treatment plan optimization in which the inverse technique is used and 2) use of intensity-modulated radiation beams. Intensity-modulated beams can be delivered either in a static fashion as a series of many irregular subfields comprising a complex intensity pattern or in a dynamic fashion by using sophisticated dynamic multileaf collimation to map a radiation-intensity profile precisely. The multileaf collimator consists of movable rectangular tungsten leaves, which are 3 to $10 \mathrm{~mm}$ in width (Fig. 1), and are mounted on a linear accelerator (Varian, Palo Alto, CA; Fig. 2). Each leaf is motorized and can move independently of the other leaves. The motion of each leaf is computer controlled. As a leaf enters a given radiation portal, it absorbs photons and casts a "shadow." The longer a leaf stays in a portion of the field, the greater the effect of its shadow. Leaves can be positioned to conform to any given outline that a target lesion might present (collimation). If the leaves are manipulated while the radiation beam is "on" (dynamic collimation), then some parts of the field will receive more radiation

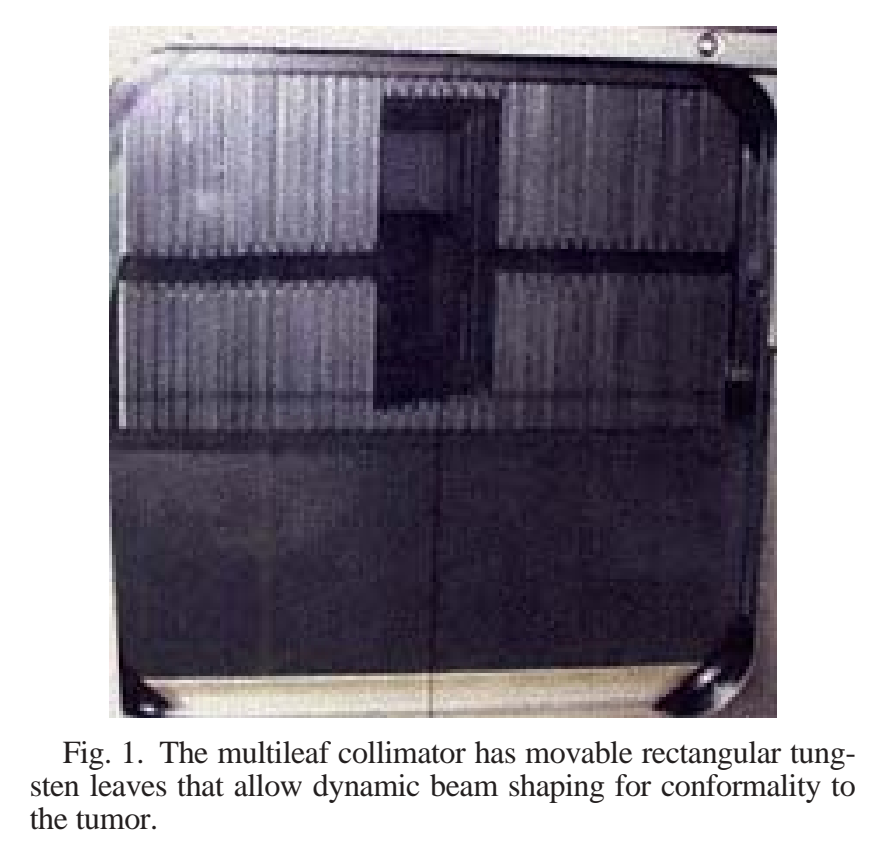

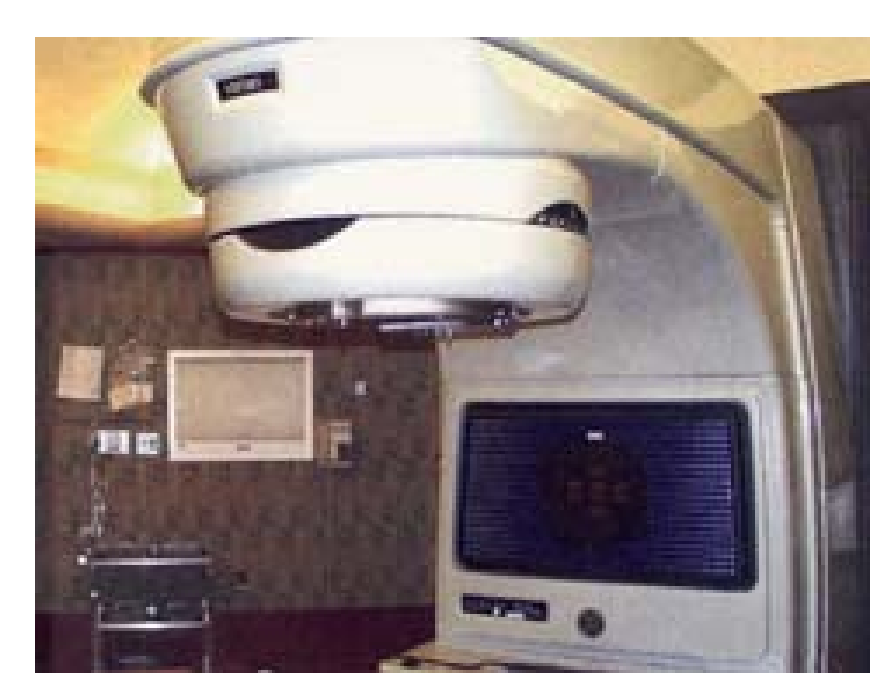

Fig. 2. The Varian linear accelerator is linked to an inverse treatment planning station, and the circular collimator is mounted on the treatment gantry for IMRT.

than other parts. Furthermore, the total time that the leaves cast a shadow will also change or modulate the dose given under each leaf. The intensity of each beam is modulated according to the demands of the 3D radiation of the target (high dose) and critical structures (low dose). Similar to intracranial stereotactic radiosurgery, multiple radiation beams create a relatively sharp dose gradient. This will allow a high dose of radiation to be adminstered to a target that may effectively wrap around the spinal cord, while the cord may receive less than $20 \%$ of the prescribed tumoral dose.

Intensity-modulated radiation therapy represents stateof-the-art treatment planning, but it is only as accurate and safe as allowed by certain physical parameters such as imaging for target/critical structure localization as well as patient immobilization and setup. Minimizing these potential sources of error is critical to optimizing the dosimetric advantages of IMRT in treating paraspinal lesions. Stereotactic techniques allow accurate localization of target lesions. As with stereotactic radiosurgery in cases of intracranial lesions, it is essential that the target be reliably identified in 3D space and no significant movement occur. Thus, paraspinal or vertebral body lesions without significant epidural extension are best suited for extracranial stereotactic techniques because of the inherent immobility of tumors affixed to the spine.

\section{Patient Immobilization}

Noninvasive stereotactic body frames have been developed to allow for reliable localization of the paraspinal lesions with an error of less than $2 \mathrm{~mm}$. At MSKCC, patient immobilization for CT-guided radiotherapy relies on a stereotactic body frame in which several pressure points are used to guarantee a reliable rigid intraprocedural immobilization of the appendicular and axial skeleton. The basic principle of immobilization is the application of pressure to osseous points, which include the lateral and anterior pelvis, lateral ribs, axilla, and sternum. The custom-made body molds emphasize the lumbar lordosis, shoulders, and neck. A patient's arms are placed above the 
head, and the legs and feet (with knees bent) are also locked into a long lower mold to control the rotation of the femoral heads. The frame has a removable anterior and fixed posterior CT-localization fiducial plate that establishes an independent coordinate system to obtain accurate anatomy localization. The calculated stereotactic coordinates can be set on a frame ruler system that includes longitudinal rulers on both sides of the frame and an arcshaped ruler for lateral and anteroposterior dimensions.

\section{Target Localization and Planning}

Treatment planning for IMRT is CT based but also relies on MR imaging and online portal images. Eligible patients undergo both CT and MR imaging while in the immobilization frame. The digital MR images can be fused with CT-based images to optimize image quality in locating target lesions and critical structures.

Once an acceptable radiation plan has been devised, daily implementation must be precise. Careful quality assurance checks are necessary to correct any deviation in positioning prior to the delivery of radiation. The immobilization frame's system can be evaluated by online portal imaging and verified using concurrent infrared camera motion studies to confirm the reliability of patient and target positioning. Online portal imaging has proven especially useful in verifying positioning in patients with metallic spinal implants. These devices can be used as reliable landmarks upon which comparisons can be made and measured.

Digitally reconstructed beam's eye-view radiographs created at the time of treatment planning (simulation x-ray films) are used as a basis of comparison with online portal images obtained prior to each treatment. These images are overlaid, digitally analyzed, and can be used to determine whether any systematic setup error is present. Necessary corrections in the $\mathrm{X}, \mathrm{Y}$, and $\mathrm{Z}$ axes can be made prior to initiation of treatment, ensuring that the isocenter of treatment is exactly correct in three-dimensional space.

\section{Treatment Data}

The IMRT program has been operational for 1.5 years at MSKCC. We have completed treatment in six patients with paraspinal tumors. Treatment data are summarized in Table 1. Tumor histology was variable, but all tumors were considered relatively radioresistant to standard doses of external-beam radiation. All patients underwent tumor resection, and three underwent multiple resections for locally recurrent tumor. In one patient nine resections were performed to treat a locally recurrent C5-6 solitary fibrous tumor. Four patients had previously undergone externalbeam radiotherapy (range 3000-4000 cGy). The indication for IMRT was local recurrence in five patients and radiation boost to the resection cavity in the sixth.

Fractionation schedules and total doses were determined by the known radiosensitivity of the tumor at extraspinal sites as well as prior radiation doses to the port. The doses ranged from $1600 \mathrm{cGy}$ in eight fractions (in cases of melanoma) to $6660 \mathrm{cGy}$ in 37 fractions (in a case of chondrosarcoma). No patient suffered acute toxicity or neurological worsening. The follow-up period has been too short to assess tumor control rates or the development of radiation-induced myelopathy.

\section{CASE ILLUSTRATION}

In 1992 this 72-year-old woman (Case 1; Table 1) underwent gross-total resection of an intermediate-grade leiomyosarcoma located in the retroperitoneum, followed by consolidation radiotherapy (to a total dose of 5040 cGy). In 1997, she presented with a metastatic tumor to T7-8 resulting in high-grade spinal cord compression and a Brown-Séquard syndrome (American Spinal Injury Association Grade C). She underwent emergency posterolateral decompression and made excellent neurological recovery (American Spinal Injury Association Grade E); thereafter, she underwent external-beam radiotherapy (to a total dose of $3000 \mathrm{cGy}$ fractions delivered in 10 doses). A number of bone metastases subsequently developed that received local radiation doses. In February 2000, she developed radicular pain, and imaging studies demonstrated a $3.5-\mathrm{cm}$ paraspinal recurrence involving the $\mathrm{T}-8 \mathrm{rib}$ and extending to the neural foramen and pedicle at T7-8 (Fig. 3 ). The patient elected to undergo IMRT rather than reresection.

The treatment plan is shown in Fig. 4. The tumor received $2000 \mathrm{cGy}$ in four fractions with $100 \%$ isodose delivered to the tumor and $20 \%$ to the spinal cord. At 1 year after IMRT the tumor was well controlled and there was no evidence of radiation-induced myelopathy. The patient has experienced significant pain relief.

\section{CONCLUSIONS}

In cases that require the delivery of high-dose radiation to paraspinal tumors, IMRT may improve local tumor control while keeping morbidity rates low. Careful treatment planning, reliable and consistent patient setup achieved using stereotactic immobilization body frames, and effective quality assurance measures are essential elements of safe and practical treatment of paraspinal lesions. Intensity-modulated radiation therapy represents a significant advance in our ability to treat both previously irradi-

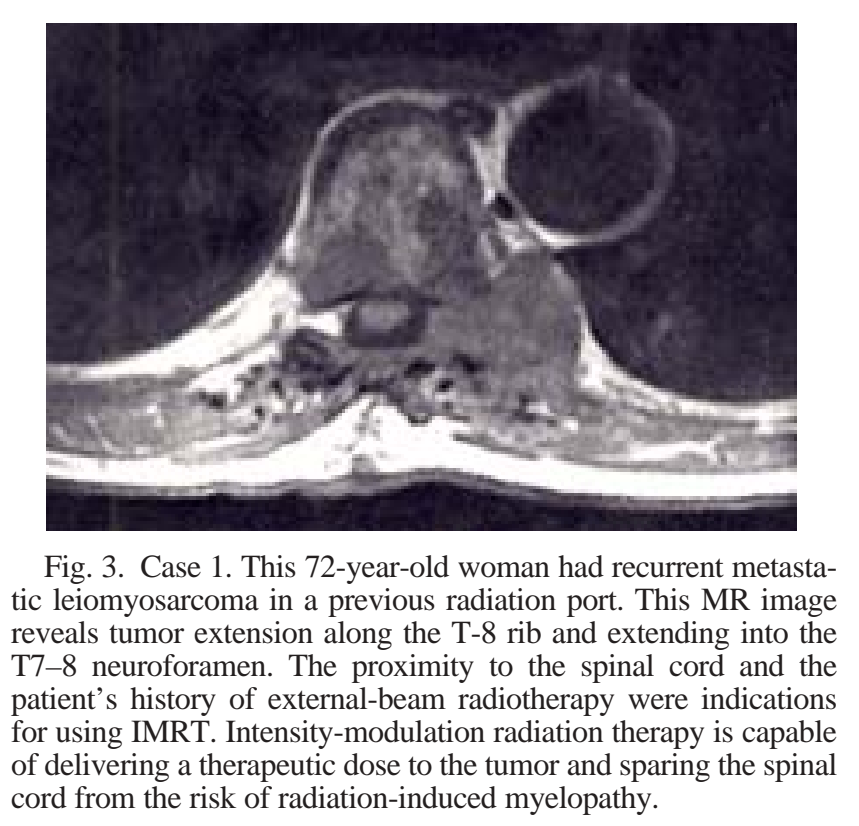




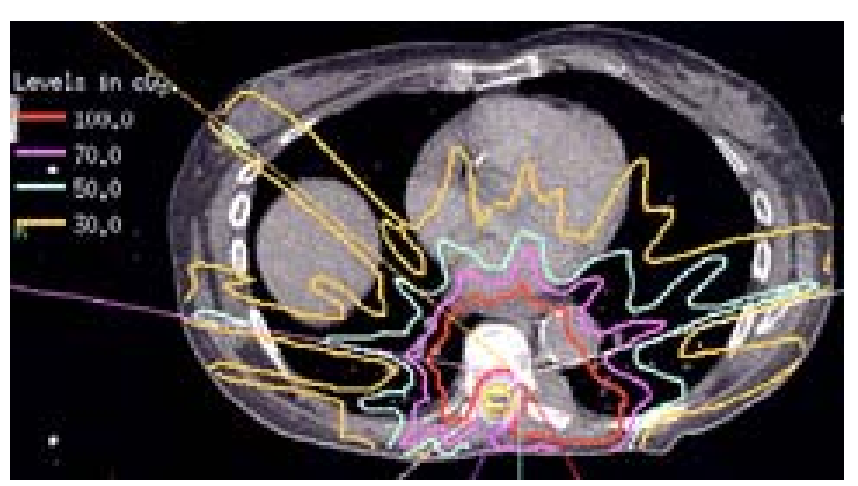

Fig. 4. Isodose lines show the percentage of the prescribed tumor dose to regions around the spine. The tumor and vertebral body received $100 \%$ of the prescribed tumor dose (2000 cGy) and the spinal cord received $20 \%$ (400 cGy), which was well within the limits of what the spinal cord could tolerate. Through dynamic beam shaping and dose delivery, IMRT is capable of creating and delivering the complex geometrical shape of the $100 \%$ isodose volume. After 1.5 years of follow up, the tumor is well controlled and there is no evidence of recurrence.

ated tumors (to a level tolerated by the spinal cord) and relatively radioresistant tumors. In the future, it may be the standard radiotherapy offered for both primary and metastatic tumors.

\section{References}

1. Ang KK: Radiobiology of the central nervous system, in Berstein M, Berger MS (eds): Neuro-Oncology. New York: Thieme, 2000, pp 160-168

2. Fehlings MG, Rao SC: Spinal cord and spinal column tumors, in Bernstein M, Berger MS (eds): Neuro-Oncology. New York: Thieme, 2000, pp 445-464

3. Hamilton AJ, Lulu B, Stea B, et al: The use of gold foil wrapping for radiation protection of the spinal cord for recurrent tumor therapy. Int J Radiat Oncol Biol Phys 32:507-511, 1995

4. Posner JB: Neurologic Complications of Cancer. Philadelphia: Davis, 1995, pp pp 111-142

5. Posner JB: Neurologic Complications of Cancer. Philadelphia: Davis, 1995, pp 311-337

6. Seichi A, Kondoh T, Hozumi T, et al: Intraoperative radiation therapy for metastatic spinal tumors. Spine 24:470-475, 1999

7. Zelefsky MJ, Fuks Z, Happersett L, et al: Clinical experience with intensity modulated radiation therapy (IMRT) in prostate cancer. Radiother Oncol 55:241-249, 2000

Manuscript received October 24, 2001.

Accepted in final form November 11, 2001.

Address reprint requests to: Mark H. Bilsky, M.D., Division of Neurosurgery, Box 71, Memorial Sloan-Kettering Cancer Center, 1275 York Avenue, New York, New York 10021. email: bilskym @mskcc.org. 